\title{
Metadata: MARS multiple stressors and biological dataset of Drava \& Mura Basins
}

Rafaela Schinegger, Christiane Aschauer, Helena Mühlmann \& Stefan Schmutz
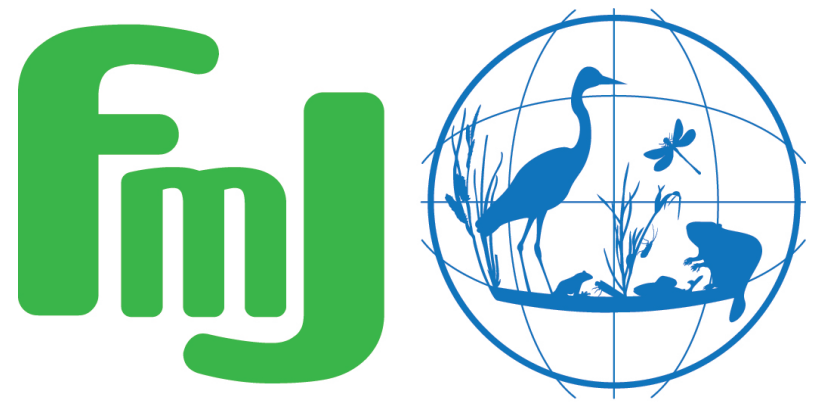

Freshwater Metadata Journal 



\title{
Metadata: MARS multiple stressors and biological dataset of Drava \& Mura Basins
}

\author{
Rafaela Schinegger ${ }^{1}$, Christiane Aschauer ${ }^{2}$, Helena Mühlmann ${ }^{1}$ \& Stefan Schmutz ${ }^{1}$ \\ 1 Institute of Hydrobiology and Aquatic Ecosystem Management, Vienna, Austria; corresponding author: \\ rafaela.schinegger@boku.ac.at \\ 2 Austrian Federal Ministry of Agriculture, Forestry, Environment \& Water Management, Vienna, Austria
}

Please cite this paper as follows: Schinegger R., Aschauer C., Mühlmann H. \& Schmutz S., 2017. Metadata: MARS multiple stressors and biological dataset of Drava \& Mura Basins. Freshwater Metadata Journal 24: 1-8. https://doi.org/10.15504/fmj.2017.24

Received: 2016-05-03 / Published: 2017-08-17

\section{Keywords}

Water Framework Directive, Stressors, Fish Assemblages, Fish Index Austria, Drava Basin, Mura Basin, Impacts, Ecological Status, Hydromorphology, MARS Project

\section{Short description of the dataset/summary}

This work/dataset addresses human stressors and their impacts on fish assemblages in the Austrian Drava and Mura River Basins. It supports the EU-project MARS (Managing Aquatic ecosystems and water Resources under multiple Stress) by analysing single and multiple stressors, environmental effects and stressor combinations/interactions. Data sources are mainly shape files and MS ACCESS databases.

With the help of point data on connectivity disruptions (barriers) and line data on hydromorphological \& water quality stressors (on water body level), six mainly hydromorphological stressors from the national inventory assessment of the EU Water Framework Directive were recoded and aggregated into new variables, i.e. stressor metrics. These then were compared with point data (fish sampling sites) and related information on fish assemblages (Fish Index Austria and related single metrics as well as the WFD biological and total status).

\section{General information}

dataset entry ID:

name of the dataset:

full name of the dataset:

dataset short name:

type of dataset:

data type:

\section{MARS_01}

Metadata: MARS multiple stressors and biological dataset of Drava \& Mura Basins

MARS DRAVA/MURA stressors and biological dataset species (taxonomic group) per site database including environmental information

point data/observation data 
science keywords according to GCMD:

topic:

keywords:
Biosphere, Biological Classification, Terrestrial Hydrosphere

Fish assemblages, metrics, rivers, stressors, impacts, ecological status, Water Framework Directive, Fish Index Austria, hydromorphological alterations, barriers

ISO topic category according to ISO 19115:

\section{Technical and administrative specifications}

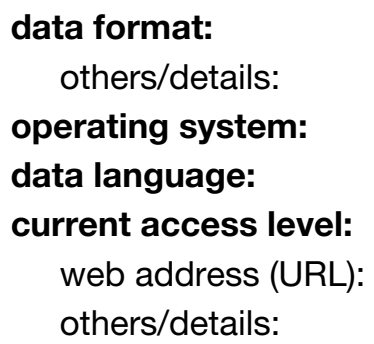

others/specify

shapefiles and MS ACCESS database

all operating systems

German

restricted access

http://www.bmlfuw.gv.at/en.html

River Basin Management Data are public data, but have to be requested from the Austrian Federal Ministry of Agriculture, Forestry, Environment and Water Management (see comment below).

no

no

no

Do you plan to publish the data on the Freshwater Biodiversity Data Portal:

comments:

\section{update level:}

others/details:

\section{documentation:}

type:

language:

others/details:

contact details:

metadata contact person:

first, last name:

phone:

email:

institution:

address:

postal code, city:

province, state:

country no

River Basin Management Data (including stressor information and biological quality element monitoring sites) are public data, but have to be requested from the Austrian Federal Ministry of Agriculture, Forestry, Environment and Water Management (see http://www.bmlfuw.gv.at/en.html).

The same applies for data on the Fish Index Austria, which have to be requested from the Institute for Water Ecology, Fish Biology and Lake Ecology (IGF; see http://www.baw.at/index.php/igf-home.html). update planned

River Basin Management Plan data from 2009 and 2015 available (2015 only as draft), next planning circle will be finished in 2021

others/specify

German

http://wisa.bmlfuw.gv.at

Helena MÃ²/4hlmann

+431711007158

helena.muehlmann@bmlfuw.gv.at

Austrian Federal Ministry of Agriculture, Forestry, Environment \& Water

Management

Marxergasse 2

1030 Vienna

Vienna

Austria 
web address:

technical contact person:

first, last name:

phone:

email:

institution:

address:

postal code, city:

country:

web address:

scientific contact person:

first, last name:

phone:

email:

institution:

address:

postal code, city:

country:

web address: http://www.bmlfuw.gv.at/en.html

Helena MÃ $1 / 4$ hlmann

+431711007158

helena.muehlmann@bmlfuw.gv.at

Austrian Federal Ministry of Agriculture, Forestry, Environment \& Water

Management

Marxergasse 2

1030 Vienna

Austria

http://www.bmlfuw.gv.at/en.html

Rafaela Schinegger

+4314765481216

rafaela.schinegger@boku.ac.at

Institute of Hydrobiology and Aquatic Ecosystem Management, BOKU Vienna Gregor Mendel Straße 33

1180 Vienna

Austria

https://www.boku.ac.at/en/personen/person/46C937474D6EB1AB/

\section{Intellectual property rights and citation}

\section{dataset creator (data compiler):}

\section{contact name:}

contact email:

contact institution:

\section{Rafaela Schinegger}

rafaela.schinegger@boku.ac.at

Institute of Hydrobiology and Aquatic Ecosystem Management (IHG)

\section{data contributors to/owners of this dataset:}

number:

multiple

2

\section{data contributor/owner 1:}

contact name:

contact email:

contact institute:

\section{Helena Mühlmann}

helena.muehlmann@bmlfuw.gv.at

Austrian Federal Ministry of Agriculture, Forestry, Environment and Water Management

criteria for using this part of the dataset:

The dataset needs to be requested from dataset creator with specific conditions of use.

\section{data contributor/owner 2:}

contact name:

contact email:

contact institute:

\section{Brigitte Sasano}

brigitte.sasano@baw.at

Institute for Water Ecology, Fish Biology and Lake Ecology (IGF) Scharfling

criteria for using this part of the dataset:

The dataset needs to be requested from dataset creator with specific conditions of use.

citation of this dataset:

author(s):

title:

year:
Schinegger, R., Aschauer, C., Mühlmann, H., Schmutz, S.

MARS stressor and biological dataset on Drava \& Mura River Basins (Austria) 2016 


\section{citation of the metadata:}

author(s): $\quad$ Schinegger R., Aschauer C., Mühlmann H. \& Schmutz S.

title and journal (name, number, pages):

Metadata: MARS multiple stressors and biological dataset of Drava \& Mura

Basins. Freshwater Metadata Journal 24: 1-8

year:

2017

doi:

https://doi.org/10.15504/fmj.2017.24

\section{dataset related references:}

reference 1:

author(s):

BMLFUW - Bundesministerium für Land- und Forstwirtschaft, Umwelt und Wasserwirtschaft

title:

Nationaler Gewässerbewirtschaftungsplan 2015 - Entwurf.

http://wisa.bmlfuw.gv.at/fachinformation/ngp/ngp-2015.html

year:

2015

reference 2:

author(s):

BMLFUW - Bundesministerium für Land- und Forstwirtschaft, Umwelt und Wasserwirtschaft

title:

Nationaler Gewässerbewirtschaftungsplan 2009 - NGP 2009

(BMLFUW-UW.4.1.2/0011-I/4/2010)

https://www.bmlfuw.gv.at/wasser/wasser-oesterreich/plan_gewaesser_ngp/nati onaler_gewaesserbewirtschaftungsplan-ngp/ngp.html

year:

2010

\section{General data specifications}

\section{regional coverage of the dataset:}

scale of the dataset: regional

continents:

Europe

spatial extent (bounding coordinates):

southernmost latitude $\left[^{\circ}\right]^{\circ}: \quad 46.6408$

northernmost latitude [ $\left[^{\circ}\right]:$

westernmost longitude [ ${ }^{\circ}$ ]: $\quad 12.92196$

easternmost longitude $\left[{ }^{\circ}\right]$ : $\quad 14.94884$

minimum altitude: $\quad 237$ metres

maximum altitude: $\quad 3798$ metres

countries: $\quad$ Europe: Austria

comments: Environmental information based on entire Drava/Mura basins in Austria.

world climatic regions according to Köppen:

Group H: alpine climates

freshwater ecoregions of the world (FEOW) according to WWF:

Europe: Central \& Western Europe

European ecoregions according to Illies (WFD):

ecosystem type:

covered timeframe:

comments:
Alps (ER4), Dinaric Western Balkan (ER5)

rivers

$2006-2015$

Timeframe of biotic data 2006-2014, abiotic/stressor data from Austrian River Basin Management Plans 2009 and 2015. 


\section{Site specifications}

coordinate system/grid data:

datum (e.g. WGS84):

grid data available: latitude/longitude, format: DD

projected, local others: MGI Lambert

31287

no

\section{ecosystem type classification:}

rivers (classification according to WFD):

altitude typology

high: $>800 \mathrm{~m}$, mid-altitude: 200 to $800 \mathrm{~m}$

exact altitudinal data available

size typology based on catchment area

small: $<100 \mathrm{~km}^{2}$, medium: $100-1000 \mathrm{~km}^{2}$

exact catchment size data available

geology

calcareous, siliceous

site coding:

site coding available:

yes, alphanumerical

number of digits:

9

example:

number of sites:

ATDRAU738

$100-1000$

exact number of sites:

525

\section{Climate and environmental data}

\section{climate related data: \\ environmental data:}

no data available

available parameters per catchment:

catchment size

data source: IHG database

presence of barriers/dams/reservoirs (fragmentation)

data source: River Basin Management Plans 2009 \& 2015

hydrological regime/flow regime

data source: River Basin Management Plans 2009 \& 2015

available parameters per site: information on embankment (incl. information on modification) data source: River Basin Management Plans 2009 \& 2015 information on channel form (incl. information on modification) data source: River Basin Management Plans 2009 \& 2015

information on cross section (incl. information on modification) data source: River Basin Management Plans 2009 \& 2015 information on water uses (e.g., irrigation, fish ponds) data source: River Basin Management Plans 2009 \& 2015

distance to next migration barrier upstream data source: River Basin Management Plans 2009 \& 2015

distance to next migration barrier downstream data source: River Basin Management Plans 2009 \& 2015

distance to the next lake upstream data source: River Basin Management Plans 2009 \& 2015

river length

data source: River Basin Management Plans 2009 \& 2015 
distance to source

data source: River Basin Management Plans 2009 \& 2015

distance to mouth

data source: River Basin Management Plans 2009 \& 2015

stream order (according to Strahler)

data source: River Basin Management Plans 2009 \& 2015 slope

data source: IHG database

altitude

data source: IHG database

hydrological regime/flow regime

data source: River Basin Management Plans 2009 \& 2015

information on instream habitat (incl. information on modification)

data source: River Basin Management Plans 2009 \& 2015

physico-chemistry data:

other physico-chemical parameters:

Chemical status available from River Basin Management Plans 2009 \& 2015.

Toxic substances available from River Basin Management Plans 2009 \& 2015.

comments:

Detailed physico-chemistry data available upon request from the Federal

Ministry of Agriculture, Forestry, Environment and Water Management.

stressors influencing the sites:

reference sites available:

\begin{tabular}{l|l|l|l|l} 
stressor & $\begin{array}{l}\text { restored sites } \\
\text { available }\end{array}$ & $\begin{array}{l}\text { data before/after } \\
\text { restoration } \\
\text { available }\end{array}$ & $\begin{array}{l}\text { stressor gradient } \\
\text { available }\end{array}$ & comments \\
& &
\end{tabular}

\begin{tabular}{|c|c|c|c|c|}
\hline eutrophication & no & no & yes & chemical status (WFD) \\
\hline
\end{tabular}

\begin{tabular}{|l|l|l|l|l}
\hline hydromorphological & no & no & yes & hydromorphological status (WFD)
\end{tabular}

\begin{tabular}{|l|l|l|l|}
\hline degradation & & \\
\hline organic pollution & no & no & yes \\
\hline
\end{tabular}

\begin{tabular}{|l|l|l|l}
\hline toxic stress & no & no & no \\
\hline
\end{tabular}

\begin{tabular}{|l|l|l|l}
\hline general degradation & no & no
\end{tabular}

\begin{tabular}{|l|l|l|l|}
\hline hydrologic stress (e.g. & no & no & yes
\end{tabular}

impoundment, flow

velocity reduction,

hydropeaking, water

abstraction, flow

velocity increase)

comments:

Various restoration studies in Upper Drava catchment conducted in the last 20 years, see http://www.life-drau.at and

Martina Humpel (2012): Metaanalyse von Eingriffen und deren

Restaurationsmaßnahmen an der österreichischen Drau. Diplomarbeit /

Masterarbeit - Institut für Hydrobiologie, Gewässermanagement (IHG),

BOKU-Universität für Bodenkultur, pp 189.

http://permalink.obvsg.at/bok/AC08907751 


\section{Biological data}

biological data origin:

specify project:

organism group addressed:

comments: from sampling

"Fish Database Austria" (FDBA, 2015), which is managed by the Institute for Water Ecology, Fish Biology and Lake Ecology (IGF) of the Federal Office of Water Management (BAW)

fish

http://www.baw.at/index.php/igf-leistungen/fischdatenbank.html

\section{Sample specifications/sample resolution}

\section{fish:}

sample information:

covered timeframe:

historical data:

palaeo data:

season:

temporal resolution/frequency of sampling:

time series data:

\section{taxonomic resolution:}

level:

percentage of species level data:

\section{taxonomic coding:}

taxalist according to:

reference(s):

coding system:

example:

\section{sample specifications:}

type:

replicate samples:

number of samples:

specification of method(s) used for sampling and sorting:

reference(s):

comments:

$2006-2014$

no

no

single date

no

family, genus, species full latin name

Thymallus thymallus

yes

25

electro fishing, wading

978-3-85174-059-2 spring, summer, autumn, winter

Leitbildkatalog (BAW IGF, 2015)

http://www.baw.at/index.php/igf-download/1693-leitbildkatalog.html

quantitative (abundance data)

Haunschmid, R., Schotzko, N., Petz-Glechner, R., Honsig-Erlenburg, W., Schmutz, S., Spindler, T., Unfer, G., Wolfram, G., Bammer, V., Hundritsch, L., Prinz, H., Sasano, B. (2010). Leitfaden zur Erhebung der biologischen Qualitätselemente Teil A1 - Fische. Bundesministerium für Land- und Forstwirtschaft, Umwelt und Wasserwirtschaft, Wien. ISBN:

http://www.baw.at/index.php/igf-download/1694-leitfaden-zur-erhebung-derbiologischen-qualitaetselemente-teil-a1-fische.html

The fish based indicators include the Fish Index Austria (FIA) and its single metrics, an IBI that was developed for the assessment of the fish-ecological status in Austria according to the WFD needs. The FIA is composed of a number of core metrics. They include number of dominant species, number of subdominant species, number of rare species, number of habitat guilds (rheophilic, limnophilic, indifferent), number of reproductive guilds (lithophilic, phytophilic, psammophilic), fish region index and population age structure of dominant and subdominant species. 


\section{Other specifications}

\section{GIS layers, shapes related to the dataset:}

catchments, river-sub-basins
dams/reservoirs/barriers
environmental variables (freshwater or terrestrial)
$\begin{aligned} & \text { availability of photos: } \\ & \text { availability of maps: } \\ & \text { quality control procedures: }\end{aligned}$ yes
Were any quality control procedures applied to your dataset?
yes
quality control protocols and comments:
Datasets were screened and data mining was conducted within a related master
thesis and within MARS WP 4 Drava basin analyses.
Aschauer, C. (2016): Distribution and patterns of multiple human stressors and
their impacts on fish assemblages in the Austrian Drava and Mura River Basins.
Master thesis, University of Natural Resources and Life Sciences, Vienna.

\section{Acknowledgements}

Sincere thanks go to the Institute for Water Ecology, Fish Biology and Lake Ecology (IGF) Scharfling, especially to Brigitte Sasano and Reinhard Haunschmid for providing Fish Index Austria data. This work was funded by the MARS project (Managing Aquatic ecosystems and water Resources under multiple Stress), funded by the European Union under the 7th Framework Programme, contract no. 603378. Finally, we are thankful to our colleague Astrid Schmidt-Kloiber for screening the metadata, for her helpful comments and for her patience regarding the publishing of this metadataset.

\section{References}

Aschauer, C., 2016. Distribution and patterns of multiple human stressors and their impacts on fish assemblages in the Austrian Drava and Mura River Basins. Master thesis, University of Natural Resources and Life Sciences, Vienna.

BMLFUW - Bundesministerium für Land- und Forstwirtschaft, Umwelt und Wasserwirtschaft, 2010. Nationaler Gewässerbewirtschaftungsplan 2009 - NGP 2009 (BMLFUW-UW.4.1.2/0011-I/4/2010)

https://www.bmlfuw.gv.at/wasser/wasser-oesterreich/plan_gewaesser_ngp/nationaler_gewaesserbewirtschaftungsplan-n gp/ngp.html

BMLFUW - Bundesministerium für Land- und Forstwirtschaft, Umwelt und Wasserwirtschaft, 2015. Nationaler Gewässerbewirtschaftungsplan 2015 - Entwurf. http://wisa.bmlfuw.gv.at/fachinformation/ngp/ngp-2015.html

Haunschmid, R., Schotzko, N., Petz-Glechner, R., Honsig-Erlenburg, W., Schmutz, S., Spindler, T., Unfer, G., Wolfram, G., Bammer, V., Hundritsch, L., Prinz, H., Sasano, B., 2010. Leitfaden zur Erhebung der biologischen Qualitätselemente Teil A1 - Fische. Bundesministerium für Land- und Forstwirtschaft, Umwelt und Wasserwirtschaft, Wien. ISBN: 978-3-85174-059-2

Humpel, M., 2012. Metaanalyse von Eingriffen und deren Restaurationsmaßnahmen an der österreichischen Drau. Diplomarbeit / Masterarbeit - Institut für Hydrobiologie, Gewässermanagement (IHG), BOKU-Universität für Bodenkultur, pp 189. http://permalink.obvsg.at/bok/AC08907751 